\title{
IMPLEMENTACIÓN Y VALIDACIÓN DE UN MATERIAL EDUCATIVO ORIENTADO A PROMOVER EL CONOCIMIENTO Y MANEJO DE LA AVULSIÓN DENTAL DE DIENTES PERMANENTES EN DOCENTES DE BÁSICA PRIMARIA Y BACHIILIERATO DE INSTITUCIONES PÚBLICAS Y PRIVADAS DE BUCARAMANGA, SANTA MARTA Y RIOHACHA
}

\author{
${ }^{1}$ Carolina Paola Brito S., ${ }^{1}$ Mónica Janeth Garnica V., ${ }^{2}$ Martha Liliana Rincón R., \\ ${ }^{3}$ Jaime Omar Moreno Monsalve. \\ ${ }^{1}$ Estudiante $X$ semestre, $F$ de Odontología U. Santo Tomás. ${ }^{2}$ Odontóloga U. Santo Tomás, \\ Especialista en Endodoncia, Docente U. Santo Tomás. ${ }^{3}$ Odontólogo U. Santo Tomás, \\ Especialista en Endodoncia, Docente, U. Santo Tomás.
}

Autor responsable de correspondencia: Carolina Paola Brito S.

Dirección de correo electrónico: caropbs@gmail.com

\begin{abstract}
RESUMEN
objetivo: Evaluar el nivel de conocimiento que tienen los docentes sobre la avulsión dental antes y después de la aplicación de un material educativo relacionado con el tema.

Materiales y Métodos: Se evaluaron 176 docentes de 16 colegios públicos y privados de Bucaramanga, Santa Marta y Riohacha acerca de la avulsión dental. Se dividieron en un grupo intervenido y un grupo control. Al grupo intervenido se le entrego un material educativo sobre el manejo de la avulsión dental. Se evaluó la efectividad del material y observar si habían diferencias entre ambos grupos. Para el análisis univariado se calcularon medidas de resumen de tendencia central para las variables cuantitativas y para las variables cualitativas se analizaron con proporciones, para el análisis bivariado se empleo test de chi cuadrado y test exacto de Fisher, según la distribución de las variables.

Resultados: El 50\% (88) pertenecían al grupo de intervenidos. El 43.2\% (76) eran de la ciudad de Santa Marta, el 38.1\% (67) eran de la ciudad de Bucaramanga y el 19\% restante (33) eran de Riohacha. El conocimiento sobre el manejo de avulsión fue regular en el 70\% (123) de la población evaluada al inicio del estudio. Después de entregado el manual de avulsión, el conocimiento fue regular en el 52.8\% (93) y se observaron asociaciones estadísticamente significativas para el nivel de conocimiento $(p=<0.0001)$ al compararse según intervención. Conclusiones: Se determinó el conocimiento y las prácticas sobre el manejo de la avulsión dental en un grupo de educadores de básica primaria y bachillerato de las ciudades de Santa Marta, Bucaramanga y Riohacha antes y después de recibir un material educativo orientado a enseñar sobre la avulsión dental, su prevención y su manejo. [Brito CP, Garnica MJ, Rincón ML. Moreno Jo. Implementación y validación de un material educativo orientado a promover el conacimiento y manejo de la avulsión dental de dientes permanentes en docentes de básica primaria y bachillerato de instituciones públicas y privadas de Bucaramanga, Santa Marta y Riohacha. Ustasalud Odontología 2006; 5: 107 - 114]
\end{abstract}

Palabras clave: Conocimiento, Avulsión, Reimplantación.

LEVEL OF KNOWLEDGE THAT YOU/THEY HAVE THE EDUCATIONAL, ONES OF BASIC PRIMARY AND HIGH SCHOOL OF INSTITUTIONS PUBLISHES AND PRIVATE OF SACRED MINX'S CITIES, BUCARAMANGA AND RIOHACHA BEFORE AND LATER OF THE INTRODUCTION OF AN EDUCATIONAL MATERIAL. PREPOSITION OF AVULSED TOOTH

\begin{abstract}
Objective: To evaluate the level of knowledge that you/they have the educational ones before on the dental extraction and after the application of an educational material related with the topic.

Materials and Methods: One hundred seventy six teachers were evaluated educational of 16 public and private schools of Bucaramanga, Santa Marta and Riohacha through a survey about the avulsed tooth. Then the educational ones were divided in an experimental and control group. An educational material was given to the experimental group about the handling of the avulsed tooth. It was carried out a second survey to evaluate the effectiveness of this material and to observe were differences between both groups.

Results: 176 were evaluated. When referring to the city $43.2 \%$ (76) were from Santa Marta, 38.1\% (67) were from Bucaramanga and the 19 (33) left were from Riohacha. $56.2 \%$ belonged to private educational institutions. The knowledge on avulsion was regulate in 70\% (123) of the population evaluated to the beginning of the study. After having given the manual the knowledge was better in $52.8 \%$ (93). It was found associations statistically significant for the level of knowledge $(\mathrm{p}=<0.0001)$ when being compared according to intervention. Conclusions: The present study determined the knowledge and the practices on avulsion, in a group of educators of elemental schools from Santa Marta, Bucaramanga and Riohacha before and after receiving an educational material.
\end{abstract}

Key words: Knowledge, Avulsion, Replantation.

Recibido para publicación: 14 de septiembre de 2006. Aceptado para publicación: 10 de diciembre de 2006 


\section{INTRODUCCIÓN}

La palabra trauma se desprende de la palabra accidente, que de acuerdo a la Real Academia Española, se define como "suceso eventual o acción del que involuntariamente resulta daño para las personas o cosas". A su vez, el término traumatismo se define como "el conjunto de lesiones del revestimiento cutáneo que interesan un tejido, un órgano o un segmento de miembro, provocado accidentalmente por un agente externo", y el termino dentoalveolar, se ajusta a la relación de los componentes adyacentes al diente/ hueso alveolar del sistema estomatognático. ${ }^{1}$

Algunos autores afirman que la prevalencia del trauma dentoalveolar en incisivos primarios y caninos es del 35.5\%, $37.8 \%$ en los niños y $32.8 \%$ en las niñas. También, se ha sugerido que los niños sufren más traumas que las niñas con una relación de 2:1. El maxilar superior se muestra más afectado en un 95.5\%. Por otra parte, el diente más afectado es el incisivo central superior $(80.5 \%)$, seguido del incisivo lateral (15\%) y por último el incisivo central inferior $(4.1 \%)$.

En Colombia, el 16.7\% de los pacientes que acuden a los servicios de urgencias odontológicas reportan fracturas dentales. Ias lesiones de los dientes se presentan, con mayor frecuencia, en el grupo de 7 a 15 años de edad. ${ }^{1,45}$

De acuerdo con la Organización Mundial de la Salud, en su clasificación de las lesiones dentales, avulsión es el desplazamiento completo del diente fuera de su alvéolo. ${ }^{6}$ Según Donaldson y Kinirons, el término avulsión dental es utilizado para describir una situación en la que, después de un trauma, un diente ha estado fuera de su alvéolo y por ende, las fibras del ligamento periodontal y el paquete neurovascular del ápice de la raíz se separan lo que conlleva a daños al alvéolo y al diente. ${ }^{7}$

Algunos estudios han reportado que la incidencia de los dientes avulsionados varía de 1 al 16\% entre todas las lesiones traumáticas de los permanentes, y de 7 al $13 \%$ para la dentición primaria. ${ }^{8,9}$ Los incisivos centrales superiores son los dientes que con mayor frecuencia son avulsionados en ambas denticiones. El género masculino experimenta más avulsiones que el femenino. ${ }^{5}$ Según Sheroan y Roberts, la avulsión dental ocurre en un $7.6 \%$ entre todas las lesiones traumáticas en dentición permanente y afecta el segmento anterior del maxilar superior. ${ }^{10}$

El pronóstico de un diente avulsionado depende del estado de las células del ligamento periodontal en el momento de la reimplantación. Por lo tanto, el manejo inmediato de un diente avulsionado puede ser definitivo para lograr la viabilidad de las células. Dicha viabilidad depende del tiempo extra-oral y del medio de almacenamiento del diente avulsionado. ${ }^{5}$

Estudios epidemiológicos han mostrado que la mayoría de los accidentes dentales ocurren en casa, seguidos del colegio. Se ha reportado que el $60 \%$ del trauma dental ocurre por un accidente escolar. Debido a que los deportes se han implicado como etiología del trauma dental y que una alta proporción de las lesiones dentales ocurre durante las clases de educación fisica, se ha sugerido que los profesores de esta área deben capacitarse en el manejo de las lesiones dentales. ${ }^{11}$

En un estudio realizado en Brasil, se encuestaron 60 docentes de colegios de básica primaria, acerca del manejo que tienen éstos en casos de avulsión dental, reveló unas cifras que muestran la falta de conocimiento acerca del manejo del trauma, principalmente, de la avulsión. ${ }^{12}$

El objetivo de este estudio fue diseñar y evaluar un medio educativo dirigido a profesores de básica primaria y bachillerato orientado a enseñar sobre la avulsión dental, su prevención y su manejo.

\section{MATERIALES Y MÉTODOS}

Se realizó un estudio basado en un estudio de tipo experimental ensayo clínico controlado. Los docentes se dividieron en forma aleatoria en un grupo experimental y un grupo control. Se realizó la entrega de un material educativo sobre el tema de interés al grupo intervenido, al grupo control no se les hizó entrega del mencionado material.

Para la muestra se tuvo en cuenta un nivel de confianza de 95\%, un poder del 80\%; relación expuestos - no expuestos $1: 1$; un conocimiento de avulsión en las personas expuestas al material educativo de un $30 \%$ y personas no expuestas, un $10 \%$; riesgo relativo 3; OR de 3.86; la muestra estuvo integrado por 142 sujetos, considerando un potencial de pérdida del $20 \%$, por lo que la muestra definitiva fue de 176 docentes. Para escoger la muestra se realizó un muestreo multietápico, se escogieron 16 colegios distribuidos de forma equitativa en cada ciudad, y de éstos, mediante una selección aleatoria se escogieron 11 docentes en cada colegio, desde primero de primaria hasta undécimo, un docente por cada grado.

Criterios de inclusión:

- Docentes que laboren en la zona urbana de las ciudades de Santa Marta, Riohacha, y Bucaramanga

- Docentes de los colegios públicos y privados de la zona urbana de las ciudades anteriormente mencionadas

- Docentes de primaria y bachillerato.

- Docentes de cualquier edad y género.

Criterios de exclusión:

- Docentes que no deseen ser encuestados.

Docentes que trabajen en colegios que por razones de seguridad o acceso de las encuestadoras no puedan ser evaluados.

108

Ustasalud Odontología 2006; 5:107 - 114

Brito CP. y col. 
Las variables estudiadas fueron:

Variables sociodemográficas: se recolecto el género, la ciudad, institución educativa.

- Variables de conocimiento: en estas variables se analizo si sabían el significado de avulsión dental, si habían tenido alguna experiencia de este tipo, y como manejarían un caso así.

- Variables sobre el material educativo: se analizó si habían tenido algún tipo de enseñanza sobre este tipo de lesión traumática, si les interesaría recibir consejos acerca del manejo de la avulsión dental y qué material educativo les gustaría recibir para aprender sobre el tema. Además, después de entregado el material educativo se evaluaron las percepciones que tuvieron sobre éste

Se escogieron 16 colegios distribuidos de forma equitativa en cada ciudad, y de estos, mediante una selección aleatoria se eligieron 11 docentes en cada colegio, desde primero de primaria hasta undécimo, un docente por cada grado.

A este grupo seleccionado se le realizó una primera encuesta en donde se evaluó qué tanto conocimiento tenían los docentes sobre la avulsión dental. Posterior a esa intervención, se dividió la muestra al azar en dos grupos de 88 docentes cada uno: un grupo llamado INTERVENIDO y el otro grupo CONTROL. Al grupo intervenido se le proporcionó un material educativo elaborado por las responsables de este estudio el cual fue diseñado de acuerdo a las necesidades de conocimiento que tuvieron los profesores según los resultados de la primera encuesta. Al grupo control no se le proporcionó dicho material.

Luego se aplicó otra encuesta constituida por 23 preguntas al grupo intervenido y ocho al grupo control que permitió evaluar el nivel de conocimiento y a la vez evaluar el material educativo en el grupo que lo recibió. Las preguntas del grupo control eran acerca del manejo de la avulsión dental nuevamente, a diferencia del grupo intervenido que además de realizarle preguntas acerca de avulsión también se les interrogó sobre las percepciones del material educativo. Las encuestas fueron diligenciadas en ambos grupos para asegurar que los objetivos propuestos.

La información fue procesada a través de una base de datos digitada por duplicado y verificando la digitación exportando los archivos digitados en Excel al paquete estadístico EPI INFO 6.04 y mediante la rutina valídate se corrobora la calidad de la digitación. Finalmente, se exportó al paquete estadístico STATA 8.0 para su correspondiente análisis.

Para el análisis univariado las variables se describieron según medidas de resumen según la naturaleza de cada una de las variables. Las variables edad y calificación del material educativo por ser cuantitativas se describieron con medidas de resumen de tendencia central como el promedio y mediana, y medidas de dispersión como el rango, varianza y desviación estándar. Las demás variables por ser cualitativas se analizaron con proporciones. Todo el análisis considero un nivel de error de $a=0.05$.

Para el análisis bivariado se aplicaron pruebas estadísticas según la naturaleza de las variables; para establecer asociaciones entre el tipo de intervención (variable de salida) con las variables cualitativas se aplico test de chi cuadrado y test exacto de Fisher y para establecer la relación entre la variable de salida con las variables cuantitativas se aplicó test de chi cuadrado según la distribución de las variables.

De acuerdo con la resolución N. 008430 del 4 de octubre de 1993 del Ministerio de Salud, este trabajo se clasificó como una investigación sin riesgo, comprende el desarrollo de acciones que contribuyen a la prevención y control de los problemas de salud, se respetó el principio de autonomía, se entregó el consentimiento informado, se explicaron los procedimientos, se definieron los objetivos, riesgos, beneficios y garantías, y la persona libremente pudo decidir si se vinculaba a la investigación.

\section{RESULTADOS}

Se evaluaron 176 docentes de los cuales el 50\% (88) pertenecían al grupo de intervenidos y el restante al grupo de no intervenidos. El 65.3\% (115) eran mujeres, el grupo de edad que registró mayor proporción fue mayores de 45 años con un 33.5\% (59) seguido del grupo de 36 a 45 años en el 29.65 (52). Al referirnos a la ciudad el $43.2 \%$ (76) eran de la ciudad de Santa Marta, el $38.1 \%$ (67) eran de la ciudad de Bucaramanga y el $19 \%$ restante (33) eran de Riohacha. El $56.2 \%$ pertenecían a instituciones educativas privadas.

Al analizar estas variables sociodemográficas según asignación de intervención no se observaron asociaciones estadísticamente significativas, lo que permite afirmar que los grupos eran homogéneos al analizar por estos aspectos (Tabla 1).

Al analizar sobre los aspectos relacionados con conocimientos sobre avulsión en el grupo de intervenidos y no intervenidos al ser evaluados inicialmente, no se observaron asociaciones estadísticamente significativas, lo que parece indicar que el conocimiento era similar entre los dos grupos; sin embargo al analizar después de entregar el manual se observaron asociaciones estadísticamente significativas para aspectos relacionados con: definición de avulsión dental ( $\mathrm{p}=<0.0001$ ) llevar al niño al odontólogo ( $p=0.003)$, buscar el diente $(p=0.05)$ y colocar el diente nuevamente en el alvéolo ( $\mathrm{p}=0.03$ ) (Tabla 2).

Al describir los aspectos relacionados con el reimplante y los procesos relacionados con este, es importante anotar que el $46 \%$ (80) lavarían el diente bajo la llave del agua y que el $28.5 \%$ (49) reportan no saber como proceder frente a esta situación. 
Tabla 1. Descripción de las variables sociodemográficas descritas en forma global y según momento de la intervención

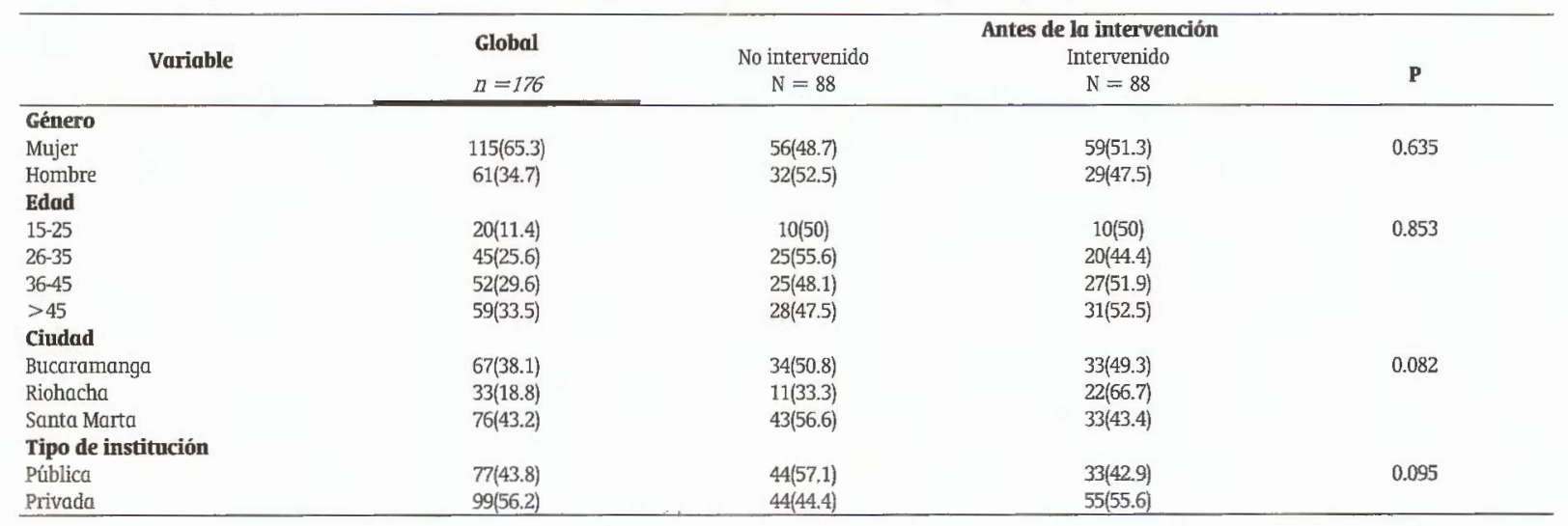

P: test de chi cuadrado $\alpha=0.05$

Al interrogar sobre el vehículo que usarían para preservar la estructura dental el 30.9\% (51) reportaron que emplearon solución antiséptica y sólo el 4.2\% (7) emplearían leche. Al evaluar inicialmente estos aspectos en los grupos que iban y no iban a ser intervenidos no se encontraron asociaciones estadísticamente significativas a diferencia de lo que se encontró después de la intervención en lo que se refiere a reimplantar el dientes $(p=0.03)$, manejo del diente sucio ( $\mathrm{p}=<0.0001$ ) y vehículo utilizado con $(\mathrm{p}=<0.0001)$. (Tabla 3)

Al analizar sobre los aspectos relacionados con la atención brindada al niño frente a la avulsión, el 44.9\% (79) de los docentes afirman que llevarían el niño al odontólogo y el $41.5 \%$ (73) el médico. La percepción sobre la urgencia de la atención frente a esta situación fue percibida por el $93.6 \%$ (161) de los sujetos evaluados. El 47.4\% (81) considera que el diente debe ser reimplantado en menores de 7 años. El comportamiento de estas variables antes y después de la intervención fue diferente pues, mientras que los resultados antes de la intervención no muestran asociaciones según asignación a tipo de intervención, en el después si se observa asociación estadísticamente significativa. Para llevar al odontólogo se observaron valores ( $\mathrm{p}=0.0001$ ) y llevar el niño al servicio de urgencias $(\mathrm{p}=0.008)$ (Tabla 4).

El conocimiento sobre el manejo de avulsión fue regular en el $70 \%$ (123) de la población evaluada al inicio del estudio. Después de entregado el manual de avulsión el conocimiento fue regular en el $52.8 \%$ (93) y se observaron asociaciones estadísticamente significativas para el nivel de conocimiento $(\mathrm{p}=<0.0001)$ al compararse según intervención (Tabla 5)

Es importante anotar que el $87.3 \%$ (151) de los docentes evaluados no han recibido ningún consejo acerca del manejo de la avulsión y el 98.8\% (170) sugieren que un manual sea el medio más adecuado para acceder a este tipo de información, destacando además que estas percepciones son coincidentes tanto en el grupo intervenido como en el grupo control (Tabla 6).
En cuanto a las percepciones del material educativo el $62.5 \%$ (55) consideró bueno el material, el 87.5\% (77) lo consideró una buena herramienta didáctica, el 72.7\% (64) consideró que el material les permitió conocer sobre el tema, el 65.5\% (57) le pareció un materia interesante y el $78.4 \%$ (69) consideran que el lenguaje permitió conocer totalmente la temática. Cerca del $91 \%$ (79) de la población intervenida afirma que el material le permitió conocer sobre la avulsión dental (Tabla 7).

En aspectos relacionados con la forma, la calificación oscilo entre 4.1 y 3.1 evaluado en puntajes entre 0 y 5 ; el primero se refiere a la secuencia de ideas que se llevó en el manual y el segundo al color del fondo lo que parece indicar una buena aceptabilidad frente al manual de avulsión (Tabla 8).

\section{DISCUSIÓN}

En la literatura revisada son muy pocos los artículos relacionados directamente con el nivel de conocimiento que tienen los docentes acerca de la avulsión dental. Sin embargo, son muchas las consecuencias que deja el mal manejo cuando ha ocurrido una lesión de este tipo; por lo tanto el mejor tratamiento es la prevención; infortunadamente, no se ha tomado muy en cuenta.

Este estudio demostró que la gran mayoría de los maestros escolares evaluados nunca ha experimentado un caso de avulsión dental. De un total de 176 encuestados de 16 colegios, el $76 \%$ no sabían el significado del termino "avulsión dental". De estos mismos docentes, el $82 \%$ nunca se había enfrentado con un caso de avulsión dental. Al igual que en el estudio realizado por Chan y colaboradores, en 2001, ${ }^{13}$ donde se evaluaron a 166 docentes de 65 colegios de los cuales el $72 \%$ no habían tenido experiencia de avulsión, los datos observados son similares a los de este estudio.

Por otra parte, Pacheco y colaboradores demostraron que en Brasil, después de realizar una encuesta para evaluar el nivel de 
Tabla 2. Descripción de las variables sobre el conocimiento de los docentes de primaria acerca de la avulsión dental en forma global y según momento de la intervención.

\begin{tabular}{|c|c|c|c|c|c|c|c|c|}
\hline \multirow[b]{2}{*}{ variable } & \multicolumn{4}{|c|}{ Antes de la intervención } & \multicolumn{4}{|c|}{ Después de la intervención } \\
\hline & $\begin{array}{c}\text { Global } \\
\mathrm{n}=176\end{array}$ & No intervenidos & Intervenidos & P & Global & No intervenidos & Intervenidos & $P$ \\
\hline \multicolumn{9}{|c|}{ Conocimiento del término avulsión dental ( $\mathrm{n}=172$ ) } \\
\hline Si & $41(28.8)$ & $19(46.3)$ & $22(52.7)$ & 0.534 & - & - & - & - \\
\hline \multicolumn{9}{|c|}{ Definición de avulsión dental (n = 173) } \\
\hline Fractura coronal & $3(1.7)$ & $1(33.3)$ & $2(66.7)$ & & $1(0.6)$ & $1(100)$ & - & \\
\hline Fractura corona raiz & $14(8.1)$ & $5(35.7)$ & $9(64.7)$ & & $12(6.9)$ & $5(41.7)$ & $7(58.3)$ & \\
\hline Diente sale de la boca & $65(35.6)$ & $32(49.2)$ & $33(50.8)$ & & $100(57.1)$ & $32(32)$ & $68(68)$ & $<0.0001$ \\
\hline No sabe & $91(52.6)$ & $48(52.8)$ & $43(47.3)$ & 0.624 & $62(35.4)$ & $49(79)$ & $13(24)$ & \\
\hline \multicolumn{9}{|c|}{ Experiencia avulsión ( $n=175$ ) } \\
\hline $\mathrm{Si}$ & $31(17.7)$ & $13(41.9\}$ & $18(58.1)$ & 0.340 & - & - & - & \\
\hline \multicolumn{9}{|l|}{ LLeva a odontólogo } \\
\hline $\begin{array}{l}\text { Si } \\
\text { Busca el diente }\end{array}$ & $103(58.5)$ & $51(49.5)$ & $52(50.5)$ & 0.878 & $122(69.3)$ & $52(46.6)$ & $70(57.4)$ & 0.003 \\
\hline $\begin{array}{l}\text { Sí } \\
\text { Llama a padres }\end{array}$ & $88(50)$ & $39(44.3)$ & $49(55.7)$ & 0.132 & $93(52.8)$ & $40(43)$ & $53(57)$ & 0.05 \\
\hline $\begin{array}{l}\text { Sí } \\
\text { Reposiciona el diente }\end{array}$ & $136(77.3)$ & $68(50)$ & $68(50)$ & 1.0 & $142(80.7)$ & $68(47.9)$ & $74(52.1)$ & 0.252 \\
\hline No sabe $|\mathbf{n}=\mathbf{1 7 5}|$ & $19(10.8)$ & $11(57.9)$ & $8(42.1)$ & 0.466 & $50(28.4)$ & $16(32)$ & $34(68)$ & 0.003 \\
\hline Si & $50(32)$ & $28(50)$ & $28(50)$ & 0.959 & $88(50.3)$ & $33(37.5)$ & $55(62.5)$ & 0.001 \\
\hline
\end{tabular}

Tabla 3. Análisis de aspectos relacionados con el reimplante de dientes descrito en forma global y según momento de la intervención.

\begin{tabular}{|c|c|c|c|c|c|c|c|c|}
\hline \multirow[b]{2}{*}{ Variable } & \multicolumn{4}{|c|}{ Antes de la intervención } & \multicolumn{4}{|c|}{ Después de la intervención } \\
\hline & $\begin{array}{l}\text { Global } \\
\mathrm{n}=176\end{array}$ & No intervenidos & Intervenidos & $\mathbf{P}$ & Global & No intervenidos & Intervenidos & $\mathbf{P}$ \\
\hline Reimplanta diente & & & & & & & & \\
\hline \multirow{2}{*}{\multicolumn{9}{|c|}{$\begin{array}{l}\text { Si } \\
\text { Diente sucio (n = 172) }\end{array}$}} \\
\hline & & & & & & & & \\
\hline Limpia con cepillo & $23(13.4)$ & $13(56.5)$ & $10(43.5)$ & & $15(0.5)$ & $\mathrm{I} 2(80)$ & $3(20)$ & \\
\hline \multirow[t]{2}{*}{ Lava con agua de llave } & $80(46.5)$ & $46(57.5)$ & $34(42.5)$ & & $118(67.1)$ & $48(40.7)$ & $70(59.3)$ & \\
\hline & & & & $0.170^{*}$ & & & & $<0.0001$ \\
\hline Reimplanta sin lavar & $4(2.3)$ & $1(25)$ & $3(75)$ & & $10(5.7)$ & $1(10)$ & $9(90)$ & \\
\hline No sabe & $49(28.5)$ & $21(42.9)$ & $28(57.1)$ & & $26(14.8)$ & $22(84.6)$ & $4(15.4)$ & \\
\hline Otro & $16(9.3)$ & $5(31.3)$ & $11(68.7)$ & & $6(3.41)$ & $5(83.3)$ & $1(16.7)$ & \\
\hline No responde & - & - & & & $1(0.6)$ & - & $1(06)$ & \\
\hline \multicolumn{9}{|c|}{ Liquido que usaría ( $n=166$ ) } \\
\hline Agua de tubo & $46(27.4)$ & $22(47.8)$ & $24(52.2)$ & & $28(15.9)$ & $23\{82.1\}$ & $5(14.4)$ & \\
\hline Leche fresca & $7(4.2)$ & - & $7(100)$ & & $42(23.9)$ & $=$ & $42(47.7)$ & \\
\hline Alcohol & $13(7.9)$ & $6(46.2)$ & $7(53.8)$ & 0.037 & $10(5.7)$ & $8(80)$ & $2(20)$ & $<0.0001$ \\
\hline Solución salina & $48(29.1)$ & $30(62.5)$ & $18(37.5)$ & & $68(38.6)$ & $30(44,1)$ & $38(55.9)$ & \\
\hline Sol. Antisépt. & $51\{30.9\}$ & $27(52.9)$ & $24\{43.1\}$ & & $28(15.9)$ & $27(96.4)$ & $1(3.6)$ & \\
\hline
\end{tabular}

P:test de chi cuadrado ( ) : exacto de fischer $\alpha=0.05$

Tabla 4. Aspectos relacionados con la atención que brindan al niño los docentes de primaria en forma global y según momento de la intervención.

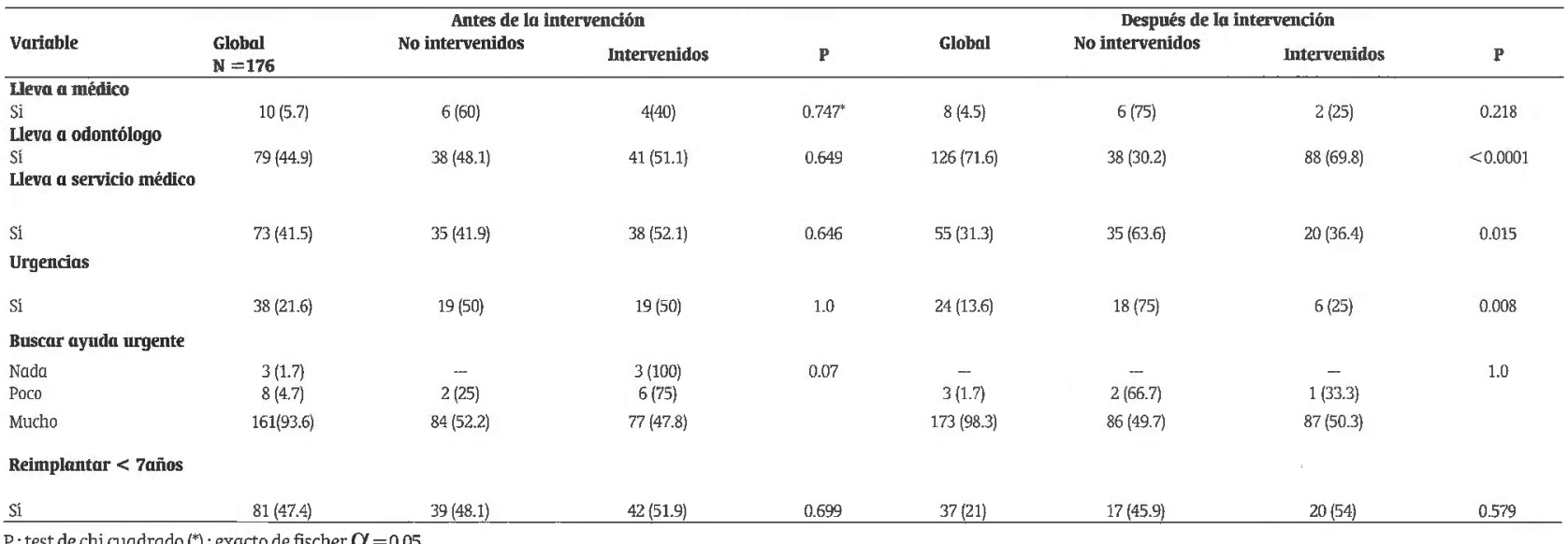


Tabla 5. Consolidado del nivel de conocimientos de los docentes de básica primaria descritos en forma global y según momento de intervención.

\begin{tabular}{|c|c|c|c|c|c|c|c|c|}
\hline \multirow[b]{2}{*}{ Variable } & \multicolumn{4}{|c|}{ Antes de la intervención } & \multicolumn{4}{|c|}{ Después de la intervención } \\
\hline & Globel & No intervenido & Intervenido & p & Global & No intervenido & Intervenuido & p \\
\hline CONOCIMIENTOS & & & & & & & & \\
\hline Malo & $38(21.6)$ & $20(52.6)$ & $18(47.4)$ & 0.368 & $20(11.4)$ & $20(200)$ & - & $<0.0001$ \\
\hline Regular & $123(69.9)$ & $58(47.2)$ & $65(52.9)$ & & $93(52.8)$ & $58(62.4)$ & 35 (37.6) & \\
\hline Bueno & $15(8.5)$ & $10(66.7)$ & $5(33.3)$ & & $63(35.8)$ & $10(15.9)$ & $53(84.1)$ & \\
\hline
\end{tabular}

P: test de chi cuadrado $\alpha=0.05$

Tabla 6. Relación entre el tipo de material educativo que le gustaría recibir según asignación a tipo de intervención.

\begin{tabular}{|c|c|c|c|c|}
\hline \multirow{2}{*}{ Variable } & \multirow{2}{*}{$\begin{array}{c}\text { Global } \\
\mathrm{N}=173\end{array}$} & \multicolumn{3}{|c|}{ Antes de la intervención } \\
\hline & & No intervenidos & Intervenidos & $\mathbf{P}$ \\
\hline \multicolumn{5}{|c|}{ Ha recibido consejos } \\
\hline No & $151(87.3)$ & $76(53.3)$ & $75(49.7)$ & 0.669 \\
\hline $\mathrm{Si}$ & $22(12.7)$ & $10(45.5)$ & $12(54.6)$ & \\
\hline \multicolumn{5}{|l|}{ Manual (n = 172) } \\
\hline No & $2(1.2)$ & $1(50)$ & $1(50)$ & 1.0 \\
\hline $\mathrm{Si}$ & $170(98.8)$ & $84(49.4)$ & $86(50.6)$ & \\
\hline \multicolumn{5}{|l|}{ Cartilla } \\
\hline No & $41(27.2)$ & $22(46.8)$ & $25(53.2)$ & 0.641 \\
\hline $\mathrm{Si}$ & $126(72.8)$ & $64(50.8)$ & $62(40.2)$ & \\
\hline \multicolumn{5}{|l|}{ Folleto } \\
\hline No & $127(73.4)$ & $65(51.2)$ & $62(48.8)$ & 0.520 \\
\hline$S \mathrm{i}$ & $46(26.6)$ & $21(45.7)$ & $25(54.4)$ & \\
\hline \multicolumn{5}{|l|}{ Plegable } \\
\hline No & $121(69.9)$ & $62(51.2)$ & $59(48.8)$ & 0.540 \\
\hline $\mathrm{Si}$ & $52(30.1)$ & $24(46.2)$ & $28(53.8)$ & \\
\hline
\end{tabular}

P: test de chi cuadrado $\alpha=0.05$

Tabla 7. Aspectos relacionados con la percepción del manual de avulsión evaluado en las personas intervenidas.

\begin{tabular}{|c|c|}
\hline Variable & $\begin{array}{c}\text { FRECUENCIA (\%) } \\
\mathbf{N}=\mathbf{8 8}\end{array}$ \\
\hline \multicolumn{2}{|l|}{ Parecer sobre el material recibido } \\
\hline Regular & $5(5.7)$ \\
\hline Bueno & $55(62.5)$ \\
\hline Excelente & $28(31.8)$ \\
\hline \multicolumn{2}{|l|}{ Manual de avulsión herramienta didáctica } \\
\hline No & $11(12.5)$ \\
\hline $\mathrm{Si}$ & $77(87.5)$ \\
\hline \multicolumn{2}{|l|}{ Material sirve para: } \\
\hline Conocer el teña & $64(72.7)$ \\
\hline Saber más del tema & $23(26.1)$ \\
\hline Sirvió poco & $1(1.1)$ \\
\hline \multicolumn{2}{|c|}{ Parecer sobre el tema del material educativo } \\
\hline Muy interesante & $28(32.2)$ \\
\hline Interesante & $57(65.5)$ \\
\hline Poco interesante & $2(2.3)$ \\
\hline \multicolumn{2}{|c|}{ Lenguaje utilizado permite comprender temática } \\
\hline Totalmente & $69(78.4)$ \\
\hline Parcialmente & $19(21.6)$ \\
\hline \multicolumn{2}{|c|}{ El manual permitió aprender sobre avuilsión dental } \\
\hline No & $8(9.2)$ \\
\hline Si & $79(90.8)$ \\
\hline \multicolumn{2}{|l|}{ El aprendizaje logrado fue } \\
\hline Poco significativo & $18(20.9)$ \\
\hline Muy significativo & $68(79.1)$ \\
\hline \multicolumn{2}{|c|}{ Es importante distribuir en todas las instituciones } \\
\hline No & $5(5.8)$ \\
\hline Si & $82(94,2)$ \\
\hline
\end{tabular}

P: test de chi cuadrado $\alpha=0.05$

Tabla 8. Calificación del manual de avulsión descrito según aspectos relacionados con la forma.

\begin{tabular}{lccc}
\hline Aspecto evaluado & Promedio \pm DE & Mediana & Rango \\
\hline Gráficos & $4 \pm 0.66$ & 4 & $2-5$ \\
Color fondo & $3.1 \pm 0.64$ & 3 & $2-4$ \\
Tamaño de letra & $3.8 \pm 0.66$ & 4 & $2-5$ \\
Color de letra & $3.8 \pm 0.55$ & 4 & 2.5 \\
Tipo de letra & $3.7 \pm 0.54$ & 4 & $3-5$ \\
Secuencia & $4.1 \pm 0.63$ & 4 & $3-5$ \\
Contenido & $3.8 \pm 0.42$ & 4 & $3-5$ \\
\hline
\end{tabular}

conocimiento sobre la avulsión dental en 60 profesores de primaria, existe poco conocimiento sobre las acciones que se deberían realizar por parte del personal docente durante la presencia de un accidente que involucre un diente avulsionado; ${ }^{12}$ se observó que el $88.3 \%$ de los docentes encuestados nunca habían tenido una experiencia con un caso de avulsión dental; otro estudio realizado en Inglaterra por Newman y colaboradores también se encontró que, de 66 docentes encuestados, el $85 \%$ no se había enfrentado con alguna experiencia de avulsión. ${ }^{14}$

Cuando se les pregunto a los docentes que harían en caso de presentarse una avulsión dental, la mayoría optó por llamar a los padres (77\%), buscar el diente para reimplantarlo ó simplemente colocarlo nuevamente; según Pacheco y colaboradores, el $57 \%$ de los docentes en Brasil llevarían al niño inmediatamente al odontólogo, el 14\% llamaría a los padres y este mismo porcentaje de docentes colocaría el diente en el alveolo y llevaría al niño al odontólogo. ${ }^{12}$

Muchos de los docentes evaluados en este estudio, al parecer contestaron las preguntas teniendo en cuenta más una base intuitiva que un conocimiento previamente adquirido, preguntas con respuestas más técnicas, por considerarse importantes para el pronóstico de la reimplantación, se contestaron a menudo inadecuadamente, por ejemplo: (1) ¿Si decidiera volver a colocar el diente en el alvéolo, pero esta sucio, Ud. que haría? y (2) ¿Que líquido usaría para lavar y transportar el diente mientras es reimplantado?

Al primer interrogante, la mayoría de los docentes (46\%) se inclinaron más por la respuesta que indicaba lavar el diente bajo un chorro de agua, respuesta comparable con el estudio realiza-

\section{2}


do en Hong Kong donde el 66\% de los docentes, que indican la mayoría, harían lo mismo; ${ }^{13}$ al igual que en Brasil, donde el $58 \%$ de los docentes actuarían de una manera similar; ${ }^{12}$ uno de los requisitos principales para el tratamiento de avulsión dental es la adecuada reimplantación del diente para mantener la viabilidad de las células del ligamento periodontal y llegar a una posible revascularización de la pulpa. La inmediata reimplantación sólo fue mencionada por el $11 \%$ de los docentes participantes en el presente estudio. Esto sugiere que los profesores no se tienen confianza para hacer el procedimiento ó simplemente no sabían como hacerlo ó si se debía hacer; exactamente igual que los docentes en Brasil, en donde sólo el 10\% pensaría en reimplantar el diente avulsionado, lo que demuestra una falta de conocimiento apropiado en la materia. ${ }^{13}$ Blakytny y colaboradores (2001) realizaron un estudio similar, encuestaron 388 maestros de 31 colegios de Estados Unidos y encontraron que el $74.5 \%$ de ellos sería incapaz de reimplantar un diente avulsionado. ${ }^{15}$

En cuanto al medio de transporte, sólo el $4 \%$ de los encuestados escogieron la leche, como la mejor elección; la mayoría, representada por el 31\%, escogieron almacenar el diente en solución antiséptica. Durante la realización del estudio en Hong Kong, a los docentes evaluados se les realizó el mismo interrogante acerca del líquido para lavar y transportar el diente; no hubo diferencias entre las respuestas dadas por los docentes de nuestro estudio comparadas con las de Chan y colaboradores porque entre los evaluados, el 5\% prefirió la leche, seguido del agua helada y soluciones antisépticas. ${ }^{13}$ Por el contrario, Blakytny y colaboradores mostraron resultados diferentes registrados en esta investigación ya que el $47 \%$ de los encuestados utilizaría como medio de transporte y almacenamiento la leche fresca. ${ }^{15}$

Se preguntó si alguna vez había recibido información sobre el manejo de la avulsión dental, a lo que la mayoría de los encuestados (87\%) contestaron que no; resultado comparable con un estudio realizado en Brasil a los estudiantes de Educación Física, en el que se observó que el $90 \%$ de los encuestados expresaron no haber recibido ningún tipo de información o consejos para el manejo de la avulsión, a lo que añadieron que consideran este asunto muy importante y necesario en especial para ellos que serán los futuros docentes que van a interactuar con niños y adolescentes durante largos períodos de tiempo. ${ }^{16} \mathrm{Al}$ igual que refiere Blakytny y colaboradores donde el $96 \%$ de los maestros expresan sus deseos por obtener información acerca de este tema. ${ }^{15}$ Esto nos muestra la necesidad urgente de educar al público con respecto al tratamiento de la avulsión dental

Después de conocer y evaluar los conocimientos de los docentes acerca de la avulsión dental y de la necesidad que tenían de una adecuada guía de manejo, se les entregó el manual titulado "SALVA TU DIENTE" Los resultados fueron los esperados, ya que el $84 \%$ (53) de los 88 que recibieron el manual mostraron tener un buen conocimiento durante la elaboración de la segunda encuesta, y lograron los objetivos propuestos al inicio de este estudio entre los cuales estaba enseñar sobre avulsión; lo que permi- te establecer comparaciones con un estudio realizado por Holan y colaboradores, en Israel, en el que compararon conocimientos, a través de una encuesta autodiligenciada, antes y después de realizar un seminario donde se incluyeron instrucciones claras en el tratamiento apropiado de la avulsión de dientes permanentes a un grupo de 126 docentes de educación física de los cuales 70 asistieron al seminario de avulsión, éstos reportaron haber adquirido un mejor conocimiento después de la charla. ${ }^{17}$

En esta investigación, a diferencia de los docentes que no leyeron el manual, los que lo hicieron demostraron la capacidad adquirida de ayudar de la mejor y más indicada manera posible en un suceso de avulsión dental. Al interrogar sobre la percepción que tuvieron del manual incluyendo el tema, el lenguaje el su utilidad, los resultados mostraron que al $62.5 \%$ (55) les pareció un material bueno y al 32\% (28) excelente, al $65.5 \%$ (57) les pareció interesante y al 32\% (28) muy interesante; el lenguaje utilizado permitió comprender la temática totalmente en el 78\% (69) de los encuestados, y el 79\% (68) reportaron un aprendizaje muy significativo,

La pérdida de los dientes permanentes es una lesión dolorosa e irreversible. Aunque la restauración de los tejidos dentales dañados hoy en día puede ser altamente efectiva, es también cierto que es muy costosa. La prevención y la educación en el manejo de traumas dentales, especialmente de la avulsión, sería entonces el mejor tratamiento.

\section{CONCLUSIONES}

El presente estudio determinó el conocimiento y las prácticas sobre el manejo de la avulsión dental en un grupo de educadores de básica primaria y bachillerato de las ciudades de Santa Marta, Bucaramanga y Riohacha antes y después de recibir un material educativo cuyo contenido estaba orientado a enseñar sobre la avulsión dental, su prevención y su manejo.

Los resultados de la primera encuesta mostraron que la mayoría de los docentes de básica primaria tuvieron un conocimiento regular sobre el tema.

El material educativo fue elaborado en forma de manual el cual se repartió en instituciones públicas y privadas a 88 docentes de básica primaria y bachillerato incluidos en el grupo intervenidos.

Luego de la implementación del manual titulado "SALVA TU DIENTE" al grupo intervenido, los resultados de la segunda encuesta mostraron que la mayoría de los docentes de primaria intervenidos tuvieron un buen conocimiento acerca del tema evaluado concluyendo así que la elaboración e implementación de dicho material lleno las expectativas planteadas en cuanto a enseñar, prevenir y manejar casos de avulsión dental.

El manual de avulsión fue aceptado en la población evaluada; a los docentes le pareció bueno ya que consideran que es una herramienta didáctica apropiada para preparar al común de la gente en 
el manejo de situaciones de avulsión dental; además sirvió para conocer el tema que, entre otras cosas, les pareció interesante y con un lenguaje que permitió comprender totalmente la temática logrando así un aprendizaje muy significativo de esta situación, concluyendo así que es de gran importancia que este material sea repartido en todas las instituciones educativas.

La implicación de este estudio incluye la necesidad de campañas al público en general sobre el manejo de la emergencia de la avulsión dental y refleja la necesidad de una mayor y más efectiva comunicación entre el personal odontológico y los profesores de las escuelas.

Finalmente, y de acuerdo con la realización de los objetivos propuestos al inicio de este proyecto, sería interesante extender el proyecto al público en general, padres de familia, niñeras, botiquines, centros recreativos, deportivos, personal médico y jóvenes estudiantes

\section{BIBLIOGRAFIA}

1. Leon JC, Contreras E, Pineda L, Galeano CF. Prevalencia del trauma dentoalveolar en pacientes atendidos en el servicio de urgencias de la clínica Carlos Ardila Lulle de Floridablanca - Colombia, entre 1998 y 2002. Ustasalud Odontología 2004; 3: 32 - 40.

2. Kramer PF, Zembmruski C, Ferreira SH, Feldens CA. Traumatic dental injuries in Brazilian pre-school children. Dent Traumatol 2003; 19: $299-303$.

3. República de Colombia. Ministerio de Salud. III Estudio Nacional de Morbilidad Oral (ENSAB III). 1998.

4. Flores MT, Andreasen JO, Bakland LK. Guidelines for the evaluation and management of traumatic dental injuries. Dent. Traumatol 2001; 17: $1-4$.

5. Ram D. Cohenca N. Therapeutic protocols for avulsed permanent teeth: Review and clinical update. Pediatr Dent 2004; 26: 251 - 255.

6. Sigalas E, Regan JD, Kramer P, Witherspoon D, Opperman L. Survival of human periodontal ligament cells in media proposed for transport of avulsed teeth. Dent. Traumatol 2004; $20: 21$ - 28.

7. Donaldson M, Kinirons MJ. Factors affecting the onset of resorption in avulsed and replanted incisor teeth in children. Dent Traumatol 2001; 17: 205 - 209.

8. Khongkunthian $\mathrm{P}$, Chantaramungkon M, Waranyuwat $\mathrm{S}$. The treatment of an avulsed maxillary central incisor by transplantation of an emvedded mandibular premolar. Dent Traumatol 2002; 18: 335 -338 .

9. Moreno Jo, Parra A. Manejo clínico del trauma dento-alveolar por el odontólogo general. Bucaramanga: Universidad Sonto Tomás, 2006. p. 12-14, 30-104.

10. Sheroan M, Mills R.. Management of a clomplex dentoalveolar trauma with multiple avulsions: a case report. Dental Traumatology 2004; 20: $222-225$.

11. Chappuis V, Von T. Replantation of 45 avulsed permanent teeth: a 1-year follow-up study. Dent Traumatol 2005; 21: $289-296$.
12. Pacheco I, Fernández F, Ferreira P, García LA, Menezes R, Villoria GEM, Ferreira SM. Evaluation of the knowledge of the treatment of avulsion in elementary school teacher in Rio the Janeiro, Brazil. Dent Traumatol 2003; 19: 76- 78.

13. Chan A, Wong T, Cheung G. Lay knowledge of physical education teachers about the emergency management of dental trauma in Hong Kong. Dent. Traumatol 2001; 17: 77 - 85.

14. Newman L, Crawford P. Dental injuries: first aid knowledge of Southampton teachers of physical education. Endod Dent Traumatol 1991; $7: 255-258$.

15. Blakytny C, Surbuts C, Thomas A, Hunter ML. Avulsed permanent incisor knowledge and attitudes of primary school teachers with regard to emergency management. Int J Paediatr Dent 2001; 11: 327 -332 .

16. Panzarini SR, Pedrini D, Brandini DA, Poi WR, Santos MF, Correa JPT, Silva FF. Physical education undergraduates and dental trauma knowledgeDent Traumatol 2005; 21: $324-328$.

17. Holan G, Cohenca N, Brin I. An oral health promotion program for the prevention of complications following avulsion: the effect on knowledge of physical education teachers. Endod Dent Traumatol 2006; 8: 160 - 162

114

Ustasalud Odontología 2006; 5:107 - 114 